\title{
DEVELOPMENT OF INFORMATION SUPPORT SYSTEM FOR THE APPLICATION OF NEW MAIZE VARIETY BASED ON SMARTPHONE
}

\author{
Feng Yang ${ }^{1}$, Shaoming $\mathrm{Li}^{1}{ }^{1, *}$ \\ ${ }^{1}$ Collage of Information and Electrical Engineering, China Agricultural University, Beijing, \\ China, 100083 \\ * Corresponding author, Address: P.O. Box 698,17 Tsinghua East Road, Haidian District, \\ Beijing, P. R. China, 100083, Tel: +86-10-82856450-8388, Fax: +86-10-82856430, Email: \\ lshaoming@sohu.com
}

Abstract: Assisted by mobile and information technologies, the risk of new maize variety application will be reduced and the efficiency will be enhanced significantly. According to the demands of new maize variety adoption and application, then an information support system which runs on Smartphone has been developed. The system consists of three main parts: information support, field problem diagnosis and business information management. Mobile development, mobile communication, mobile database and expert system technologies were used in the process of this program. The system offers a kind of new technology and approach for the new maize variety application. Moreover, it can provide powerful information support to users, and is applicable and easy-to-use.

Keywords: new maize variety application, Smartphone, mobile database, expert system

\section{INTRODUCTION}

The seed industry is now in a new period of great change in China (Sun Shixian, 2003). At present, there is a widespread phenomenon in China's seed industry that good variety has always lacked suitable method. On the

Yang, F. and Li, S., 2008, in IFIP International Federation for Information Processing, Volume 259; Computer and Computing Technologies in Agriculture, Vol. 2; Daoliang Li; (Boston: Springer), pp. 817-824. 
one hand, it is difficult to select a suitable cropping field for the new variety. Moreover, we often lack scientific cultivation methods which take actions that suit to local circumstances. On the other hand, farmers often are accustomed to cultivate all of the varieties in the same way. So it is difficult for the new variety to have a good performance and higher yield. However, maybe there is a reduction in output (Zhu Jianfang, 2001). For the rapid conversion of new varieties to productivity, it is necessary to cultivate the new variety in a suitable and scientific way. The informationalization of the new variety adoption and application will play a crucial role to the scientific application of new variety.

The major works of the new variety application are carried out in the field in China's rural areas, but computer and Internet is still in the preliminary stage of development there. So portability, simplification and economy are the basic requirements for the information products of new variety application. As a representative of mobile computer system, Smartphone is becoming popular rapidly. Combined with modern wireless communication and WAP, Smartphone has been applied to mobile commerce, mobile office, and mobile life and so on. It changed the mode of thinking in the traditional sense that the computer is only used in the office or fixed place. It also has brought about the informationalization and digitalization developing into personal application which is deep and anywhere. Since Smartphone is easy to carry, powerful, economical and other reasons, the information system based on Smartphone has become the only way of the informationalization of new variety application.

An information support system for new maize variety adoption and application based on Smartphone has been designed and developed in this paper, which used modern information technologies synthetically such as mobile development technology, mobile database technology and expert system. The system runs on the operation system of Windows Mobile 5.0. And it consists of information support, field problem diagnosis and business information management. Because it is small in size, easy to operate and economical, there will be a good prospect.

\section{OVERALL STRUCTURE AND FUNCTIONS OF SYSTEM}

The information support system for the application of new variety runs on Smartphone. It is designed against more information demands, portability and resource sharing. System is divided into three levels, including the movable terminal, server and database. The overall structure of system is 
shown in Fig. 1, including: information database, information support module, field problem diagnosis module, business information management module, information upload and download module.

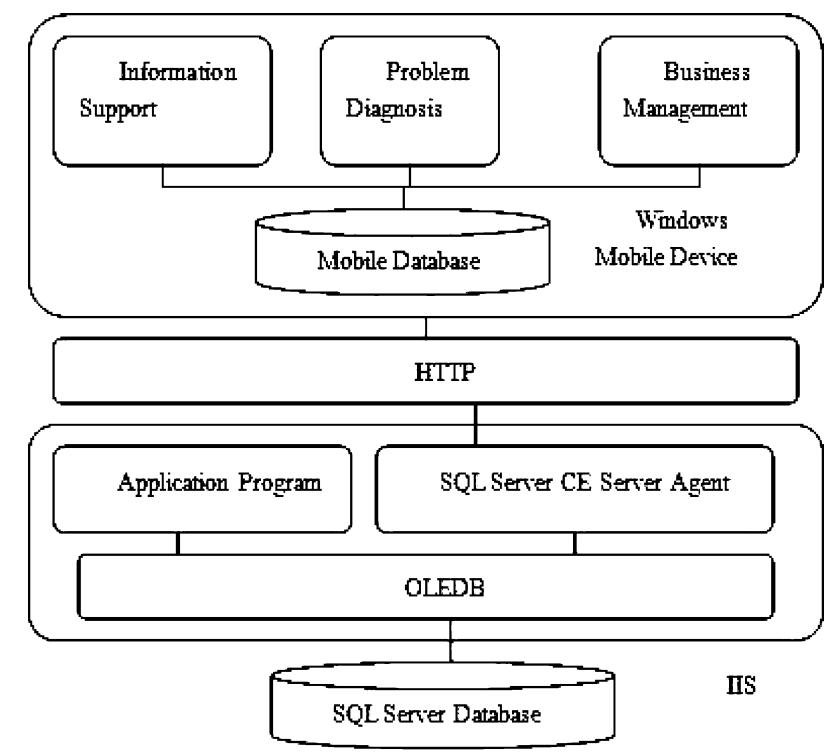

Fig. 1. The overall structure of system

Information Database: There are many kinds of and large quantities of information for the system. But the storage space and data processing capacity of Smartphone are limited, it is necessary to establish a database system including server database and mobile terminal database. When network is connected, Smartphone is used as a client of server through a wireless network, and completes data processing and maintenance by taking advantage of the mature database technology of PC platform; otherwise, Mobile database can ensure that the system is used in the off-line state, and also can record the updated and added information on the mobile terminal. Database mainly stores three kinds of information: one is used for inquiry, including variety information, county's environment information, dealers' information and business information; the other is used for field problem diagnosis, including expert knowledge, maize disease information, nutrition lack information and cultivation information; and another is survey information, including planting growing environmental investigation information, agronomic management survey information, varieties performance investigation information, competitive species survey information, and seed market survey information. 
Information support module: It mainly implements adding, modifying, inquiring and deleting information on varieties, county's environment information, cultivation habits, variety preference and dealer information. There are two ways for inquiring: On the one hand, it implements inquiries in line with the input information. On the other hand, combined with a digital map, it implements inquiries according to the chosen region on the digital map.

Problem diagnosis module: It mainly provides cultivation management, disease diagnosis and nutrition lack diagnosis. According to the description of problem from farmer, it implements disease diagnosis and nutrition lack diagnosis by using the cases of problem diagnosis in the knowledge base, and then offers diagnosis outcome, prevention and cure measures.

Business management module: It consists of main two parts: daily business management, which provides adding, modifying, deleting and inquiring information on daily business in a variety of ways, and special investigation which supports the growing environmental investigation, agronomic management survey, varieties performance investigation, competitive species survey, seed market survey, the key agricultural files and so on.

Upload and download module: For maize planting, it is a prerequisite to take actions that suit to local circumstances. So every region requires suitable varieties. Because the storage space of Smartphone is limited, it is necessary to download information according to different region, and update information to the Information Center.

\section{KEY TECHNOLOGY RESEARCH}

\subsection{Problem Diagnosis Technology}

\subsubsection{Knowledge Representation}

It is the basis of problem diagnosis that the experts' knowledge is represented correctly and stored effectively. According to the characters of development platform, this program structures the knowledge of maize diseases and nutrition lack in terms of the object-oriented knowledge representation (Yang Zhong, 2003): First, identifying the object. To identify all objects, the research problem should be analyzed in detail. If some objects have the similar character, a special class, which is defined as lower class, should be created by abstracting these similar objects. Then, if the 
lower classes have the similar character, a parent class should be created by abstracting these similar objects. It is should be going on until there is no common. Moreover, all objects should be defined and named. Finally, there will be a knowledge base model of the problem field. Taking maize diseases for example, at first, the common attribute of disease place should be classified into root, stem, leaf, fruit four main categories according to the symptoms of the disease. In four major categories, then the common of the objects' symptoms should be abstracted respectively, such as lesion or lesion place, color and so on. The common character should be abstracted and summarized gradually until the final diagnosis of various diseases.

\subsubsection{Inference Engine Implementation}

Problem diagnosis module is an expert system virtually. The way used for reasoning is forward inference. The basic idea of this reasoning is that, starting from the known facts, a conclusion is reasoned out by using positive rules (Gao Chunming, 2002). To reason, the module requires moralized rules and man-machine dialogue interface which adapted the requirements of Smartphone. Taking maize disease for example, the module diagnoses the maize diseases, nutrition lack by reasoning mechanism, and gives the corresponding prevention and cure methods. As follows:

if $($ diseaseSite $==$ "leaf"

and spotsAndModuleStateMembranes = = "moiré, brown bacteria"

and earlySymptoms $==$ "oval lesion appeared on leaves"

and lateSymptoms $==$ "lesions expand into moiré; they are grass green in the central of lesion, grayish brown on the edge; and there are white and brown small bacteria tumors; leaves even wither when serious");

According to the expert knowledge, the conclusion is shown in the Fig. 2.

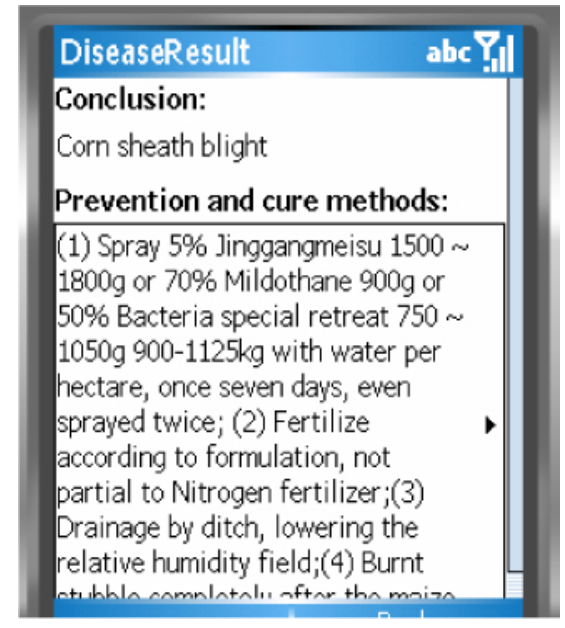

Fig. 2. The diagnosis result 


\subsubsection{Knowledge Base Maintenance}

It is ideal for expert system to learn and preserve knowledge themselves. But the capacity of Smartphone is limited (Ouyang Jianquan, 2002). So it is difficult for the expert system runs on Smartphone to learn and preserve knowledge themselves. However, it is clever to maintain knowledge base by a distributed way. That is, the database used on Smartphone could be created on PC firstly; or the database on PC could be converted to the database that Smartphone requires. By this way, the knowledge database on Smartphone could be updated by the knowledge database on PC when Smartphone could communicate with PC. Advantages are that it could take advantage of the mature database technology on PC and avoid maintaining the knowledge database in a small screen; moreover, the interface is much friendlier. At the same time, the resources of PC and Smartphone are fully exploited. In addition, they could use the resources of each other.

\subsection{Mobile Database Technology}

With the popularization of mobile terminals, there are higher demands for the real-time processing and management to mobile data. Mobile database is increasingly showing its advantages. For resource constraints, mobile equipments are used as the front-end application together with general application system. The data sets on the mobile equipment may be the subset of the data set on back-end server or the copy of the subset on the server. Generally, mobile database keeps synchronous with the database server through some data replication mode (upload, download or mixed mode). Thus meet the demands that people visit arbitrary data in arbitrary location and at any time (Zhou Shumin, 2007).

Web Service data synchronization, Replication and Remote Data Access (RDA) are three main data synchronization methods (Zhou Shumin, 2007):

1) Web Service data synchronization is a functional set which is packed into a single entity published on the Internet and available for other procedures. 2) Replication, SQL Server Mobile replication is based on Microsoft SQL Server replication. Mobile equipment and database server exchange data automatically by replication. Replication could provide a resolution to the data conflict mechanism. 3) Remote Data Access (RDA) permits applications access the data of SQL Server database from the remote, and store the data in the local SQL Server Mobile database table. The table of SQL Server table could be updated by application procedures according to the changes of local records (Liu Yanbo, 2006). 
Data on server are downloaded by SQL Server Mobile applications running on mobile equipment through RDA. In the system, the database, which based on Windows Mobile 5.0, is designed with SQL Mobile and SQL Server 2005 development environment. And data synchronization is implemented through RDA.

\section{DEVELOPMENT AND IMPLEMENTATION OF SYSTEM}

Smartphone, which supports wireless communication and network connection, is used as hardware. The system runs on the operation system of Microsoft Windows Mobile 5.0, and based on the Microsoft .NET Compact Framework 2.0. And it is implemented with C \# and Microsoft Visual Studio 2005. Database management platform is SQL Mobile.

\section{CONCLUDING REMARK}

In this paper there is a desk study on the design and implementation of the information support system for the application of new maize variety based on Smartphone. According to the business characteristic of the new variety application, the system provides a solution program against the problems of immediate inquiry and resources sharing in the process of new variety application. Mobile development, mobile communication, mobile database and expert system technologies were used in the process of this program. Application of information technology and mobile technology, the program has been designed and implemented to assist strong and updatable information for the application of new maize variety. Thus, the new good variety and suitable cultivation methods are offered together. It is likely that the new variety will have a good performance. So the risk of new crop variety application will be reduced and the efficiency will be enhanced significantly. With the development of Smartphone, mobile communication, the Internet and other technologies, information systems based on Smartphone in the variety industry would have a wider application in the near future.

\section{ACKNOWLEDGEMENTS}

This research is funded by the Support of Science and Technology Project of the State, Programmed award No. 2006BAD10A01 from March 2007 to November 2009. 


\section{REFERENCES}

Gao Chunming, Chen Yuexin, Su Liang, Liu Dongbo. Direct Reason-Machine System Research. Computer Engineering \& Application. 2002, (19):78-80.

Guo Yinqiao, Guo Xinyu, Li Cundong, Zhao Chuande, Zhao Chunjiang. Knowledge modelbased decision support system for maize management. Transactions of the Chinese Society of Agricultural Engineering. 2006, 22(10):163-166.

Liu Yanbo, Hu Yanyan, Ma Ji. Windows Mobile Application and Development Platform. Beijing: POST \& TELECOM PRESS. 2006, 336-339.

Ouyang Jianquan, Qian Queliang, Chu Chengyuan, Li Jintao. The Design and Implement of PDA-Oriented Expert System in Agriculture. Computer Engineering \& Application. 2002, (2):30-31.

Peng Hai-yan, Chen Gui-fen, Peng Hai-ying, Yang Lian-hui, Tong Mei. Study and development of expert systemdiagnosis of maize about the scathe for disease and worm. Computer and Agriculture. 2002, (3):16-17.

Sun Shixian. Forecast for the generalization of new varieties during "tenth-five". Seed Science \& Technology. 2003, (3):127-128.

Yang Zhong, Zuo Hongfu, Shen Chunlin. Realization of Object-Oriented Rule-Type Expert System Template. Transactions of Nanjing University of Aeronautics \& Astronautics. 2003, 20(2):218-223.

Zhou Shumin, Zhang Tiantai, Xu Zhiwen. The Data Synchronization of Embedded Database and Its Application]. Control \& Automation. 2007, 23(5-2):79-80.

Zhu Jianfang, Yang Xianzhong, Wang Yunhua. Accelerate the pace of new varieties, promote the development of seed industry. Seed. 2001, (3):78-79. 